\title{
LA COMISIÓN NACIONAL DE LOS DERECHOS HUMANOS COMO AUTORIDAD RESPONSABLE PARA EFECTOS DEL JUICIO DE AMPARO
}

\author{
THE NATIONAL HUMAN RIGHTS COMMISSION AS RESPONSIBLE AUTHORITY \\ FOR PURPOSES OF AMPARO TRIAL \\ VERÓNICA CERVERA TORRES ${ }^{1}$ \\ SYLVIA TORRES CABALLERO²
}

Resumen: La Comisión Nacional de los Derechos Humanos (CNDH) ha sufrido importantes transformaciones a lo largo de su vida institucional que la han dotado de mayores facultades y autonomía, sin embargo, ha sido poco explorado si la CNDH puede ser considerada como autoridad responsable para efectos de procedencia del juicio de amparo y por ende, susceptible de cometer violaciones a derechos humanos cuando paradójicamente su objetivo principal es velar por la protección no jurisdiccional de los mismos. El presente artículo expondrá brevemente el origen y evolución en el diseño constitucional de esta institución a lo largo de sus 29 años de existencia y revisará diversos criterios jurisdiccionales que dan cuenta del cambio de visión, particularmente a partir de las reformas en materia de amparo y derechos humanos del año 2011, que conllevan a considerar a la CNDH como autoridad responsable para efectos del juicio de amparo.

Palabras Clave: Comisión Nacional de los Derechos Humanos; ombudsman; amparo.

${ }^{1}$ Licenciada en Derecho por la Universidad La Salle; Especialidad en Derechos Humanos y Maestría en Derecho por la Universidad Nacional Autónoma de México; Maestría en Derechos Humanos y Democracia por la Facultad Latinoamericana de Ciencias Sociales; Doctorante en el Instituto de Investigaciones Jurídicas de la Universidad Nacional Autónoma de México

${ }^{2}$ Licenciada en Derecho, Maestría en Derecho y Doctorado en Derecho por la Universidad Nacional Autónoma de México; se ha desempeñado como abogada litigante en materias laboral, mercantil y familiar. Actualmente labora en la Comisión Nacional de los Derechos Humanos. 
Abstract: The National Human Rights Commission (CNDH) has undergone important transformations throughout its institutional life that have endowed it with greater powers and autonomy; however, it has been little explored if the CNDH can be considered as the responsible authority for the purposes of origin of the amparo judgment and therefore, capable of committing human rights violations when paradoxically its main objective is to ensure the non-legal protection of the same. This article will briefly explain the origin and evolution in the constitutional design of this institution throughout its 29 years of existence and will review various jurisdictional criteria that account for the change in vision, particularly from the reforms in the area of amparo and rights of 2011, which entails considering the CNDH as the responsible authority for the purposes of the amparo proceeding.

KEYwords: National Human Rights Commission; ombudsman; amparo.

SUMARIO: I. Introducción; II. Origen y evolución del diseño constitucional de la CNDH; III. Reforma constitucional en materia de amparo de 2011; IV. La CNDH como autoridad responsable para efectos del juicio de amparo; V. Conclusiones; VI. Fuentes.

\section{I N T RO D U CCIÓ N}

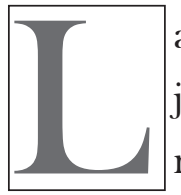

a Comisión Nacional de los Derechos Humanos (CNDH) junto con sus homólogas en las entidades federativas, conforman el sistema no jurisdiccional de protección a derechos humanos en México cuyo función radica en la protección de los derechos humanos, de conformidad con el artículo 102, apartado B) de la Constitución Política de los Estados Unidos Mexicanos. Pese a que sus determinaciones no revisten carácter vinculatorio a diferencia del sistema jurisdiccional, resulta viable considerar a la CNDH como autoridad para efectos del juicio de amparo a partir de las reformas en materia de derechos humanos y amparo de 2011 que posibilitan situar a la CNDH como posible transgresora de derechos humanos. Para comprender esta transición, en el primer apartado se revisará brevemente el origen y evolución 
de la CNDH como organismo desconcentrado, organismo descentralizado y organismo constitucional autónomo; en el segundo apartado las modificaciones con motivo de la reforma en materia de amparo de 2011; en el tercer apartado se expondrán diversos criterios jurisdiccionales que reflejan el cambio de criterio para considerar a la CNDH como autoridad responsable para efectos del juicio de amparo y finalmente en las conclusiones se reflexionará acerca del mandato inexcusable de la CNDH de no transgredir derechos humanos y cumplir con sus funciones en favor de los mismos o de lo contrario, estar sujeta a un control de tipo jurisdiccional.

\section{ORIGEN Y EVOLUCIÓN DEL DISEÑO CONSTITUCIO-} NAL DE LA CNDH

La figura ombudmsan de origen sueco ${ }^{3}$ (actualmente mejor referida como ombudsperson bajo el enfoque de perspectiva de género) hace referencia a un vocero, representante o mediador de la ciudadanía ${ }^{4}$; que a lo largo del tiempo fue implementada en varios países bajo diversas modalidades y matices ${ }^{5}$ aunque conservando como esencia el fiscalizar la actividad administrativa de las autoridades públicas mediante procedimientos sencillos y rápidos ${ }^{6}$.

\footnotetext{
${ }^{3} \mathrm{Al}$ respecto, Véase Sonia Venegas Álvarez, Origen y devenir del Ombudsman ${ }_{\mathrm{C}}$ una institución encomiable?, México, Universidad Nacional Autónoma de México-Instituto de Investigaciones Jurídicas, 1988, p. 11.

${ }^{4}$ Donald C. Rowat, El ombudsman. El defensor del ciudadano, México, Fondo de Cultura Económica, 1973, p. 7.

${ }^{5}$ Sobre el tema, Véase Héctor Fix Zamudio, Protección Jurídica de los Derechos Humanos. Estudios Comparativos, México, Comisión Nacional de los Derechos Humanos, 1991, p. 347-394

${ }^{6}$ Héctor Fix Zamudio, Los derechos humanos y su protección jurídica y procesal en Latinoamérica, p. 17.
} 
El carácter autónomo de esta institución, entre otras cualidades, se considera esencial a su naturaleza de conformidad con los "Principios relativos al Estatuto y Funcionamiento de las Instituciones Nacionales de Protección y Promoción de los Derechos Humanos”, también conocido como "Principios de París", documento adoptado por la Asamblea General de las Naciones Unidas en $1993^{7}$.

En nuestro país si bien algunos autores destacan como antecedentes de esta figura a la Dirección para la Defensa de los Derechos Humanos en Nuevo León de 1979; el Procurador de Vecinos en Colima de 1983; la Procuraduría para la Defensa del Indígena en Oaxaca de 1986; la Procuraduría Social de la Montaña en Guerrero de 1987; la Procuraduría de Protección Ciudadana en Aguascalientes de 1988; la Defensoría de los Derechos de Vecinos en Querétaro de 1988; la Defensoría de los Derechos Universitarios de 1985 y la Procuraduría Social del Departamento del Distrito Federal de 1989'; fue hasta el 13 de febrero de $1989^{9}$ que se creó una Dirección General de Derechos Humanos dentro de la Secretaría de Gobernación, entre cuyas funciones se encontraban proponer programas, proyectos de iniciativas, acciones, capacitaciones y mecanismos en la materia; proporcionar apoyo técnico y asesoría sobre instrumentos internacionales a derechos humanos; así como atender quejas administrativas sobre violación de derechos humanos.

${ }^{7}$ Véase Oficina del Alto Comisionado de las Naciones Unidas para los Derechos Humanos, Instituciones Nacionales de Derechos Humanos. Antecedentes, principios, funciones y responsabilidades, Nueva York y Ginebra, Naciones Unidas, 2010, pp. 37-64.

${ }^{8}$ José Luis, López Chavarría, et. al., Evolución normativa de la Comisión Nacional de Derechos Humanos, México, 1993, p. 11.

${ }^{9}$ Véase Reglamento Interior de la Secretaría de Gobernación, publicado el 13 de febrero de 1989 en el Diario Oficial de la Federación, disponible en: http://www.dof. gob.mx/nota_to_imagen_fs.php?cod_diario $=206855$ \&pagina $=26 \&$ seccion $=0$ 
Posteriormente, el 6 de junio de $1990^{10}$ se creó por decreto presidencial la CNDH como un órgano desconcentrado de dicha Secretaría, responsable de proponer y vigilar el cumplimiento de la política nacional en la materia; con funciones de establecer mecanismos de coordinación adecuados; ejecutar programas de atención y seguimiento en torno a los reclamos sociales de derechos humanos; proponer programas preventivos y acciones que impulsaran el cumplimiento de instrumentos internacionales de derechos humanos, así como la posibilidad de formular recomendaciones sobre violaciones de derechos humanos.

Fue hasta la reforma publicada en el Diario Oficial de la Federación el 28 de enero de $1992^{11}$, que se le dotó de rango constitucional mediante la adición de un apartado B) al artículo 102 de la Constitución Política de los Estados Unidos Mexicanos, estableciendo a la CNDH como un organismo descentralizado, además de conformar un sistema no jurisdiccional de protección a los derechos humanos, al prever que las legislaturas de los estados también establecerían estos organismos en cada entidad federativa que conocerían de quejas en contra de actos $\mathrm{u}$ omisiones de naturaleza administrativa provenientes de cualquier autoridad o servidor público (con excepción de los del Poder Judicial de la Federación, asuntos electorales, laborales y jurisdiccionales) que violaran derechos humanos y reiterando la facultad para formular recomendaciones públicas autónomas, no vinculatorias y denuncias y quejas ante

${ }^{10}$ Véase Decreto por el que se crea la Comisión Nacional de Derechos Humanos como un órgano desconcentrado de la Secretaría de Gobernación, publicado el 6 de junio de 1990 en el Diario Oficial de la Federación, disponible en: http://www. dof.gob.mx/nota_to_imagen_fs.php? codnota $=4659530 \&$ fecha $=06 / 06 / 1990 \&$ cod_ diario $=200653$

${ }^{11}$ Véase Decreto por el que se reforma el Artículo 102 de la Constitución Política de los Estados Unidos Mexicanos, publicado el 28 de enero de 1992 en el Diario Oficial de la Federación, disponible en: http:/www.dof.gob.mx/nota_to_imagen_fs.ph p? codnota $=4646760 \&$ fecha $=28 / 01 / 1992 \&$ cod_diario $=200014$ 
las autoridades respectivas. También se estableció la facultad de CNDH para conocer de las inconformidades que se presentaran en relación con las recomendaciones, acuerdos u omisiones de los organismos equivalentes a las entidades federativas.

Otra importante modificación constitucional al mismo apartado, sucedió con la reforma de 13 de septiembre de $1999^{12}$, ya que consolidó a la CNDH como un organismo constitucional autónomo con autonomía de gestión y presupuestaria, así como personalidad jurídica y patrimonio propios. Asimismo, estableció un Consejo Consultivo y estableció la obligación del titular de la CNDH de presentar anualmente un informe de actividades a los Poderes de la Unión.

Como otra relevante transformación, debemos mencionar a la reforma en materia de derechos humanos de 10 de junio de $2011^{13}$, la cual introdujo otra serie de modificaciones en el artículo y apartado en comento, a saber:

1. Establece que todo servidor público se encuentra obligado a responder las recomendaciones formuladas por estos organismos y en el caso de que no sean aceptadas o cumplidas, las autoridades o servidores públicos deberán fundar, motivar y hacer pública su negativa y además, la Cámara de Senadores o en sus recesos la Comisión Permanente o las legislaturas de las entidades federativas, según corresponda, podrán llamar a solicitud de estos organismos, a las autoridades o servidores

${ }^{12}$ Véase Decreto por el que se reforma y adicional el artículo 102 apartado B de la Constitución Política de los Estados Unidos Mexicanos, publicado el 13 de septiembre de 1999 en el Diario Oficial de la Federación, disponible en: http:/www.dof.gob.mx/ nota_to_imagen_fs.php?

${ }^{13}$ Véase Decreto por el que se modifica la denominación del Capítulo I del Título Primero y reforma diversos artículos de la Constitución Política de los Estados Unidos Mexicanos, publicado el 10 de junio de 2011 en el Diario Oficial de la Federación, disponible en: http://www.dof.gob.mx/nota_to_imagen_fs.php? codnota $=5194486 \& f e c$ ha=10/06/2011\&cod_diario=237901 
públicos responsables para que comparezcan ante dichos órganos legislativos a fin de que expliquen el motivo de su negativa.

2. Habilita la competencia para conocer de asuntos electorales y jurisdiccionales.

3. Mandata que las constituciones de las entidades federativas y el entonces Estatuto de Gobierno de la Ciudad de México deberían garantizar la autonomía de los organismos de protección de los derechos humanos.

4. Modifica la elección del titular de la CNDH y sus homólogas en las entidades federativas, así como de los integrantes del consejo consultivo, al sujetarlo a un procedimiento de consulta pública transparente.

5. Habilita la competencia de la CNDH para investigar hechos que constituyan violaciones graves de derechos humanos, cuando lo juzgue conveniente o lo pidiera el Ejecutivo Federal, algunas de las Cámaras del Congreso de la Unión o los titulares de los poderes ejecutivos de las entidades federativas o las legislaturas de éstas, incluidas la entonces Asamblea Legislativa del Distrito Federal.

Ahora bien, resulta pertinente mencionar que la reforma en materia de derechos humanos modificó otros once artículos ${ }^{14}$ de la Constitución Política de los Estados Unidos Mexicanos (1, 3, 11, 15, 18, 29, 33, 89, 97, 105), destacando el artículo primero constitucional al disponer que todas las personas gozarán de los derechos humanos reconocidos en esa Constitución y en los tratados internacionales de los que el Estado Mexicano sea parte, así como de las garantías para su protección, cuyo ejercicio no podrá restringirse ni suspenderse, salvo en los casos y bajo las condiciones que la propia Constitución establece. Por otra parte, refiere que las normas relativas a los derechos humanos se interpretarán de conformidad con la Constitución y los tratados internacionales de la materia, favoreciendo en todo tiempo a las personas la protección más

\footnotetext{
14 Ídem.
} 
amplia y señala que todas las autoridades (que incluiría a la $\mathrm{CNDH}$ ), en el ámbito de sus competencias, tienen la obligación de promover, respetar, proteger y garantizar los derechos humanos de conformidad con los principios de universalidad, interdependencia, indivisibilidad y progresividad. Por lo que el Estado deberá prevenir, investigar, sancionar y reparar las violaciones a los derechos humanos.

La última modificación constitucional al artículo 102, apartado B) de la Constitución Política de los Estados Unidos Mexicanos fue la publicada en el Diario Oficial de la Federación el 29 de enero de $2016^{15}$, para adecuar los términos en atención al cambio de la Ciudad de México como entidad federativa.

La creación de la CNDH obedeció a múltiples causas tanto políticas, económicas y sociales que propiciaron su implementación en nuestro país ${ }^{16}$ y que actualmente es cuestionada su existencia en el contexto del actual gobierno ${ }^{17}$. No se omite mencionar que la institución ha sido objeto de anteriores críticas en torno a su funcionamiento a lo largo de su existencia ${ }^{18}$, sin embargo, se considera que la figura del ombudsperson es

15 Véase Decreto por el que se declaran reformadas y derogadas diversas disposiciones de la Constitución Política de los Estados Unidos Mexicanos en materia de la reforma política de la Ciudad de México, publicado en el Diario Oficial de la Federación el 29 de enero de 2016, disponible en: http://www.dof.gob.mx/nota_detalle.php? ${ }^{\text {codigo }}=54240438$ fecha $=29 / 01 / 2016$

16 Véase José Galindo Rodríguez, La CNDH: una consecuencia de la política económica y social de México (1970-1990), México, Porrúa, 2010, pp. 7-9.

${ }^{17}$ Víctor Chávez, Busca Morena quitar la CNDH y crear Defensoría del Pueblo, en El Financiero, México, 8 de abril de 2019, disponible en: https://www.elfinanciero.com. $\mathrm{mx} /$ nacional/busca-morena-quitar-la-cndh-y-crear-defensoria-del-pueblo

${ }^{18}$ Cfr. Francisco Javier Acuña Llamas, La Comisión Nacional de los Derechos Humanos: una institución a medio camino. Frentes críticos a su estatuto jurídico inconcluso, México, Porrúa-Universidad Anáhuac del Sur, 2003, pp. 217-252; Emilio Álvarez Icaza et. al., Ombudsman: asignatura pendiente en México, México, Universidad Iberoamericana, 2013, pp. 17-65; Human Rights Watch, La Comisión Nacional de los Derechos Humanos de México. Una evaluación crítica, Nueva York, 2008, Human Rights Watch, pp. 1-10; e Instituto Tecnológico Autónomo de México, Programa Ata- 
necesaria en un estado democrático de derecho cuyo eje sean los derechos humanos y que a la vez constituye un importante contrapeso al poder. Cobrando vigencia en la actualidad las palabras de quien fue el primer titular de ese organismo: la Comisión Nacional es un organismo del presente y del futuro ${ }^{19}$.

III. REFORMA CONSTITUCIONAL EN MATERIA DE AMPARO DE 2011

El juicio de amparo es la institución jurídica, mediante la cual, una persona denominada quejoso solicita a un órgano jurisdiccional federal el amparo y protección de la justicia federal, en contra de un acto o ley (acto reclamado), emitida u omitida por una autoridad denominada responsable y que el quejoso considera viola sus garantías individuales (hoy derechos humanos) o el régimen de distribución competencial, lo cual le causa un agravio, para que se le restituya o mantenga en el goce de sus presentes derechos ${ }^{20}$.

La reforma constitucional en materia de amparo fue publicada el 6 de junio de $2011^{21}$ y se destacan las siguientes modificaciones que se consideran relevantes para el tema de nuestro interés:

laya. Análisis académico de la CNDH y CDHDF, México, 2003, Instituto Tecnológico Autónomo de México.

${ }^{19}$ Jorge Carpizo, Derechos humanos y Ombudsman, México, 1993, Comisión Nacional de los Derechos Humanos-Instituto de Investigaciones Jurídicas de la Universidad Nacional Autónoma de México, p. 12.

${ }^{20}$ Hertino Avilés Albavera, Introducción al estudio del derecho de amparo, México, 2012, Facultad de Derecho y Ciencias Sociales de la Universidad Autónoma del Estado de Morelos, p. 65.

${ }^{21}$ Véase Decreto por el que se reforman, adicionan y derogan diversas disposiciones de los artículos 94, 103, 104 y 107 de la Constitución Política de los Estados Unidos Mexicanos, publicado en el Diario Oficial de la Federación el 6 de junio de 2011, disponible en: https://www.dof.gob.mx/nota_to_imagen_fs.php? cha $=06 / 06 / 2011 \&$ cod_diario $=237741$ 
1. Se dispuso que los Tribunales de la Federación resolverían toda controversia que se suscite: a) por normas generales, actos u omisiones de la autoridad que violen los derechos humanos reconocidos y las garantías otorgadas para su protección por esta Constitución, así como por los tratados internacionales de los que el Estado Mexicano sea parte; b) por normas generales o actos de la autoridad federal que vulneren o restrinjan la soberanía de los estados o la esfera de competencia del Distrito Federal, y c) por normas generales o actos de las autoridades de los Estados o del Distrito Federal que invadan la esfera de competencia de la autoridad federal.

2. Se señaló que el juicio de amparo se seguirá siempre a instancia de parte agraviada, teniendo tal carácter quien aduce ser titular de un derecho o de un interés legítimo individual o colectivo, siempre que alegue que el acto reclamado viola los derechos reconocidos por esta Constitución y con ello se afecte su esfera jurídica, ya sea de manera directa o en virtud de su especial situación frente al orden jurídico.

3. En el juicio de amparo deberá suplirse la deficiencia de los conceptos de violación o agravios de acuerdo con lo que disponga la ley reglamentaria.

4. En materia administrativa el amparo procede, además, contra actos $\mathrm{u}$ omisiones que provengan de autoridades distintas de los tribunales judiciales, administrativos o del trabajo, y que causen agravio no reparable mediante algún medio de defensa legal. Será necesario agotar estos medios de defensa siempre que conforme a las mismas leyes se suspendan los efectos de dichos actos de oficio o mediante la interposición del juicio, recurso o medio de defensa legal que haga valer el agraviado, con los mismos alcances que los que prevé la ley reglamentaria y sin exigir mayores requisitos que los que la misma consigna para conceder la suspensión definitiva, ni plazo mayor que el que establece para el otorgamiento de la suspensión provisional, 
independientemente de que el acto en sí mismo considerado sea o no susceptible de ser suspendido de acuerdo con dicha ley.

Con motivo de esta reforma, sufrió modificaciones la Ley de Amparo, reglamentaria de los artículos 103 y 107 de la Constitución Política de los Estados Unidos Mexicanos, regulando las causas de improcedencia del juicio de amparo en el artículo $61^{22}$.

Las adecuaciones anteriores, sumado a lo dispuesto en el artículo primero, tercer párrafo, de la Constitución Política de los Estados Unidos Mexicanos que revisamos en apartado anterior, permite vislumbrar a la CNDH como autoridad responsable para efectos de amparo en caso de ser transgresora de derechos humanos que ampara el orden jurídico mexicano y por tanto, susceptible de un control de tipo jurisdiccional a través de este mecanismo.

\section{LA CNDH COMO AUTORIDAD RESPONSABLE PARA EFECTOS DEL JUICIO DE AMPARO}

Han sido pocos los criterios jurisprudenciales que denotan un cambio de criterio a fin de considerar a la CNDH como autoridad responsable para efectos de amparo, mismos que se exponen a continuación:

1.- Comisión Nacional de Derechos Humanos. Es improcedente el amparo en contra de la declaratoria de incompetencia para conocer de una denuncia de violación a derechos humanos, por no ser un acto de autoridad ${ }^{23}$.

2.- Comisión Nacional de Derechos Humanos. Sus recomendaciones no tienen el carácter de actos de autoridad para efectos del juicio de

\footnotetext{
${ }^{22}$ Cfr. Artículo 61 de la Ley de Amparo, reglamentaria de los artículos 103 y 107 de la Constitución Política de los Estados Unidos Mexicanos

${ }^{23}$ Tesis aislada P. XCVII/98, Semanario Fudicial de la Federación y su Gaceta, Novena Época, t. VIII, diciembre de 1998, p. 223.
} 
$\operatorname{amparo}^{24}$.

3.- Comisión Nacional de Derechos Humanos. La negativa a expedir copia de constancias relativas a los expedientes de queja a las partes que la solicitan para exhibirlas en juicio, viola la garantía de audiencia, aun cuando se aduzcan razones de confidencialidad ${ }^{25}$.

4.- Comisión Nacional de los Derechos Humanos. El desechamiento del recurso de impugnación previsto por el artículo 55 de su ley, es un acto de autoridad para efectos de la procedencia del juicio de amparo ${ }^{26}$. 5.- Procede el juicio de amparo cuando la comisión nacional de derechos humanos desecha un recurso de impugnación por improcedente ${ }^{27}$.

De los criterios anteriores se colige que la $\mathrm{CNDH}$ en un principio no fue considerada como autoridad para efectos de amparo dado que sus resoluciones, incluidas las recomendaciones, no creaban, modificaban o extinguían una situación jurídica concreta dado su carácter de no vinculatorias.

En un segundo momento, se consideró que la negativa de $\mathrm{CNDH}$ para expedir copias de las constancias de expedientes de queja a las partes con la finalidad de exhibirlas en juicio, violaba la garantía de audiencia pese al principio de confidencialidad contenido en su propia normatividad $^{28}$ y por tanto, tal situación era susceptible de conocimiento vía juicio de amparo como una excepción a la regla anterior.

\footnotetext{
${ }^{24}$ Tesis aislada VI.3o.16 K, Semanario Fudicial de la Federación y su Gaceta, Novena Época, t. IX, abril de 1999; p. 507.

${ }^{25}$ Tesis aislada P. XLVIII/2000, Semanario fudicial de la Federación y su Gaceta, Novena época, t. XI, abril de 2000, p. 65.

${ }^{26}$ Tesis aislada IV.3ํ․A.33K, Semanario Fudicial de la Federación y su Gaceta, Décima Época, t. I, diciembre de 2014, p. 3421.

${ }^{27}$ Tesis Jurisprudencial (común) 1a./J. 23/2018, Semanario Fudicial de la Federación y su Gaceta, Décima Época, t. I, octubre de 2018, p. 716.

${ }^{28}$ Véase artículo 68 de la Ley de la Comisión Nacional de los Derechos Humanos.
} 
En un tercer momento, posterior a la reforma en materia de derechos humanos y amparo de 2011, se advirtió que el desechamiento del recurso de impugnación por parte de $\mathrm{CNDH}$ se considera como un acto de autoridad para efectos del juicio de amparo, bajo el argumento de la relación de supra a subordinación con un particular, siendo equiparable la facultad de resolver el recurso respectivo a la de un órgano jurisdiccional; siendo irrenunciable por su naturaleza pública la potestad sobre resolver los recursos presentados y cuyo ejercicio, representa un acto unilateral a través del cual extingue una situación jurídica que afecta la esfera legal de un particular que no cuenta con recurso alguno contra el desechamiento y por tanto queda en un estado de indefensión, resultando necesario examinar la legalidad de su actuación.

Asimismo, bajo el último criterio enlistado que consolidó jurisprudencia y de nuevo bajo el marco posterior a la reforma de derechos humanos y amparo expuestas; se reafirmó que la CNDH debe apegarse a los estándares de legalidad exigibles a todas las autoridades, de lo contrario, se impide el acceso a la tutela no jurisdiccional de derechos humanos y el desechamiento de un recurso de impugnación sin apego a dichos estándares, resulta en un acto intraprocesal que extingue situaciones jurídicas, de forma unilateral, obligatoria y que puede generar violaciones a derechos humanos. No obstante, se reiteró que las recomendaciones no son consideradas actos de autoridad para efectos del juicio de amparo debido a su efecto no vinculatorio y en consecuencia, no eran susceptibles de alterar la esfera jurídica de las personas.

Ahora bien, resulta importante mencionar otros casos en los cuales ha procedido el juicio de amparo contra la CNDH como el caso de la inadecuada investigación relacionada con la masacre de 72 migrantes en 
San Fernando, Tamaulipas ${ }^{29}$; sobre la negativa de investigación de la queja de un periodista ${ }^{30}$; sobre la negativa de entrega de información de un expediente de seguimiento de recomendación sobre graves violaciones a derechos humanos ${ }^{31}$; sobre la falta del pronunciamiento de la calidad de víctima en el caso Ayotzinapa ${ }^{32}$; sobre el acceso a expedientes de queja con independencia de la conclusión de su trámite ${ }^{33}$ y sobre cuestionar la falta de material probatorio para emitir una recomendación ${ }^{34}$. Con independencia de la resolución acerca del fondo de cada uno de los asuntos anteriores, se resalta que se admitió la demanda de amparo.

En consecuencia, como denominador común de los casos en los cuales la CNDH fue considerada claramente como autoridad para efectos de amparo, se advierte que fueron criterios posteriores a la reforma en

${ }^{29}$ Fundación para la Justicia y el Estado Democrático de Derecho, Tribunal colegiado confirma admisión de amparo contra CNDH en el caso de la masacre de 72 migrantes en San Fernando, México, 6 de junio de 2014, disponible en: fundacionjusticia.org/tribunal-colegiado-confirma-admision-de-amparo-contra-cndh-en-el-caso-de-la-masacre-de-72-migrantes-en-san-fernando/

${ }^{30}$ Colectivo contra la Tortura y la Impunidad, Periodista gana amparo contra CDHDF y CNDH por negativa a investigar caso de tortura, México, 2 de abril de 2018, disponible en: https://contralatortura.org.mx/index.php/component/k2/item/170-periodista-gana-amparo-contra-cdhdf-y-cndh-por-negativa-a-investigar-caso-de-tortura-abogado-defensor-solicita-medidas-cautelares-contra-mancera-y-ebrard

${ }^{31}$ Comisión Mexicana de Defensa y Promoción de los Derechos Humanos, CNDH obligada a entregar expediente de seguimiento sobre caso de violaciones graves a derechos humanos, México, 9 de octubre de 2017, disponible en: https://cmdpdh.org/2017/10/cndh-obligada-entregar-expediente-seguimiento-caso-violaciones-graves-derechos-humanos/

32 Rubén Mosso, Juez otorga amparo a jugador de Los Avispones, en Milenio, México, 30 de julio de 2018, disponible en: https://www.milenio.com/policia/juez-otorga-amparo-a-jugador-de-los-avispones

${ }^{33}$ Emir Olivares Alonso, Falla fuez contra artículo de la CNDH que vulnera garantía de acceso a la información, La Jornada, México, 6 de febrero de 2019, disponible en: https:// www.jornada.com.mx/2019/02/06/politica/010n3pol

${ }^{34}$ Redacción, Dan plazo a CNDH para documentar recomendación contra Felipe Muñoz Vázquez, El Clarinete, México, 1 de mayo de 2019, disponible en: http://www.elclarinete. com.mx/dan-plazo-a-cndh-para-documentar-recomendacion-en-su-contra/ 
materia de derechos y humanos y amparo del 2011. De los ejemplos anteriores, se advierte que la CNDH puede ser señalada por vulnerar derechos humanos tales como el derecho al acceso a la información; derecho a la seguridad jurídica; derecho de acceso a la justicia bajo el sistema no jurisdiccional de protección a derechos humanos y derechos de las víctimas por falta de debida diligencia en la investigación y reconocimiento de su calidad de víctima.

\section{CONCLUSIONES}

Es evidente que un organismo constitucional del nivel y naturaleza jurídica de la CNDH en nuestro país no debe ser ajeno a los principios básicos de legalidad y seguridad jurídica. Es decir las determinaciones de la $\mathrm{CNDH}$ pueden y deben estar sujetas a la revisión de otros órganos competentes como el poder judicial, que verifiquen la motivación y fundamentación respectiva de sus resoluciones.

Durante el presente trabajo, se dio cuenta de la evolución en el diseño constitucional de la CNDH pero a su vez del cambio de paradigma a partir de la reforma en materia de amparo y derechos humanos de 2011, que llevaron a replantear al poder judicial la posibilidad de que los actos de la CNDH constituyen actos de autoridad para efectos de amparo, considerando que tal institución aunque revista un carácter autónomo y sus determinaciones no cuenten con un efecto vinculatorio, no escapa de la obligación contenida en el artículo 1, párrafo tercero de la Constitución Política de los Estados Unidos Mexicanos, a efecto de proteger los derechos humanos y no vulnerarlos.

La transformación jurídica del ombudsperson en nuestro país ha traído grandes momentos de oportunidad para que los juristas y toda la sociedad pueda reestudiar o replantear sus actuaciones y alcances jurídicos, pues aún y cuando se trata del principal organismo encargado de la noble misión de velar por el cumplimiento de los derechos humanos, puede en ocasiones quedar atrasado, 
rebasado o bien coartado en su alcance e intenciones al cometer transgresiones a derechos humanos.

Es prioritario que en el contexto actual, la $\mathrm{CNDH}$ cumpla cabalmente con su mandato y se constituya como una auténtica defensora de derechos humanos sin incurrir en ningún tipo de violaciones, con miras a que no sea cuestionada su legitimidad y credibilidad como institución ante la sociedad y con ello, garantizar su permanencia.

\section{FUENTES}

\section{BIBLIOGRAFIA}

Álvarez IcAza, Emilio et. al., Ombudsman: asignatura pendiente en México, México, Universidad Iberoamericana, 2013.

Acuña Llamas, Francisco, La Comisión Nacional de los Derechos Humanos: una institución a medio camino. Frentes críticos a su estatuto jurídico inconcluso, México, Porrúa-Universidad Anáhuac del Sur, 2003.

Avilés Albavera, Hertino, Introducción al estudio del derecho de amparo, México, 2012, Facultad de Derecho y Ciencias Sociales de la Universidad Autónoma del Estado de Morelos,

Carpizo, Jorge, Derechos humanos y Ombudsman, México, 1993, Comisión Nacional de los Derechos Humanos-Instituto de Investigaciones Jurídicas de la Universidad Nacional Autónoma de México,

Galindo Rodríguez, José, La CNDH: una consecuencia de la política económica y social de México (1970-1990), México, Porrúa, 2010.

Human Rights Watch, La Comisión Nacional de los Derechos Humanos de México. Una evaluación crítica, Nueva York, 2008, Human Rights Watch.

Instituto Tecnológico Autónomo de México, Programa Atalaya. Análisis académico de la CNDHy CDHDF, México, 2003, Instituto Tecnológico Autónomo de México. 
López Chavarría, José Luis, López Chavarría, et. al., Evolución normativa de la Comisión Nacional de Derechos Humanos, México, 1993.

Fix Zamudio, Héctor, Protección Furídica de los Derechos Humanos. Estudios Comparativos, México, Comisión Nacional de los Derechos Humanos, 1991.

Oficina del Alto Comisionado de las Naciones Unidas para los Derechos Humanos, Instituciones Nacionales de Derechos Humanos. Antecedentes, principios, funciones y responsabilidades, Nueva York y Ginebra, Naciones Unidas, 2010.

Venegas Álvarez, Sonia, Origen y devenir del Ombudsman iuna institución encomiable?, México, Universidad Nacional Autónoma de México-Instituto de Investigaciones Jurídicas, 1988.

Rowat, Donald C., El ombudsman. El defensor del ciudadano, México, Fondo de Cultura Económica, 1973.

\section{NORMATIVIDAD}

Constitución Política de los Estados Unidos Mexicanos

Ley de la Comisión Nacional de los Derechos Humanos

Ley de Amparo, reglamentaria de los artículos 103 y 107 de la Constitución Política de los Estados Unidos Mexicanos

\section{PAginAS DE INTERNET}

Diario Oficial de la Federación

www.dof.gob.mx

Suprema Corte de Justicia de la Nación

www.scnj.gob.mx 


\section{HEMEROGRAFICAS}

Chávez, Víctor, Busca Morena quitar la CNDH y crear Defensoría del Pueblo, en El Financiero, México, 8 de abril de 2019, disponible en: https:/www.elfinanciero.com.mx/nacional/busca-morena-quitar-la-cndh-y-crear-defensoria-del-pueblo

Colectivo contra la Tortura y la Impunidad, Periodista gana amparo contra CDHDF y CNDH por negativa a investigar caso de tortura, México, 2 de abril de 2018, disponible en: https:/contralatortura.org.mx/index.php/component/k2/ item/170-periodista-gana-amparo-contra-cdhdf-y-cndh-por-negativa-a-investigar-caso-de-tortura-abogado-defensor-solicita-medidas-cautelares-contra-mancera-y-ebrard

Comisión Mexicana de Defensa y Promoción de los Derechos Humanos, CNDH obligada a entregar expediente de seguimiento sobre caso de violaciones graves a derechos humanos, México, 9 de octubre de 2017, disponible en: https:// cmdpdh.org/2017/10/cndh-obligada-entregar-expediente-seguimiento-caso-violaciones-graves-derechos-humanos/

Fundación para la Justicia y el Estado Democrático de Derecho, Tribunal colegiado confirma admisión de amparo contra CNDH en el caso de la masacre de 72 migrantes en San Fernando, México, 6 de junio de 2014, disponible en: fundacionjusticia.org/tribunal-colegiado-confirma-admision-de-amparo-contra-cndh-en-el-caso-de-la-masacre-de-72-migrantes-en-san-fernando/

Mosso, Rubén, Juez otorga amparo a jugador de Los Avispones, en Milenio, México, 30 de julio de 2018, disponible en: https://www.milenio.com/policia/ juez-otorga-amparo-a-jugador-de-los-avispones

Olivares Alonso, Emir, Falla Juez contra artículo de la CNDH que vulnera garantía de acceso a la información, La Jornada, México, 6 de febrero de 2019, disponible en: https://www.jornada.com.mx/2019/02/06/politica/010n3pol 
Redacción, Dan plazo a CNDH para documentar recomendación contra Felipe Muñoz Vázquez, El Clarinete, México, 1 de mayo de 2019, disponible en: http:// www.elclarinete.com.mx/dan-plazo-a-cndh-para-documentar-recomendacion-en-su-contra/ 